Cahiers d'études italiennes

Enea Silvio Piccolomini-Pie II

\title{
Pie II dans les œuvres poétiques et oratoires de Giannantonio Campano
}

John Nassichuk

\section{(2) OpenEdition}

Journals

Édition électronique

URL : http://journals.openedition.org/cei/87

DOI : $10.4000 /$ cei.87

ISSN : 2260-779X

Éditeur

UGA Éditions/Université Grenoble Alpes

Édition imprimée

Date de publication : 15 octobre 2011

Pagination : 173-186

ISBN : 978-2-84310-207-3

ISSN : 1770-9571

\section{Référence électronique}

John Nassichuk, «Pie II dans les œuvres poétiques et oratoires de Giannantonio Campano », Cahiers d'études italiennes [En ligne], 13 | 2011, mis en ligne le 15 avril 2013, consulté le 27 mars 2021. URL http://journals.openedition.org/cei/87 ; DOl : https://doi.org/10.4000/cei.87 


\title{
PIE II DANS LES CEUVRES POÉTIQUES ET ORATOIRES DE GIANNANTONIO CAMPANO
}

\author{
John Nassichuk \\ Université Western Ontario
}

Encore aujourd'hui, le premier titre de gloire de Giannantonio Campano demeure sa contribution à la vie culturelle du pontificat de Pie II. C'est principalement grâce aux citations récurrentes de ses épigrammes dans les Commentaires ${ }^{1}$ de ce pontife, ainsi qu'à l'importante oraison funèbre qu'il prononça lors des obsèques du pape $^{2}$, que l'humaniste originaire du Mezzogiorno se trouve encore fréquemment associé à l'entourage de son ami et bienfaiteur siennois. Nommé évêque de Crotone en I462, puis de Teramo dès l'année suivante, Campano bénéficia largement du patronage de ce pontife humaniste. Les 672 vers adressés à Pie dans l'édition principale de ses Carmina ${ }^{3}$ témoignent aussi du fait qu'il n’hésita guère à chanter la gloire - et à demander le soutien - de son patron et confrère qui l'apprécia réellement pour ses talents considérables. La critique a bien montré, à la suite des remarques de Paparelli et de Flavio di Bernardo dans sa biographie de Campano ${ }^{4}$, que celui-ci loue avec ardeur et éloquence le protecteur

I. Voir l'important article de S. De Beer, "The Pointierung of Giannantonio Campano's Epigrams: Theory and Practice ", dans S. De Beer, K. A. E. Enenkel et D. Rijser, The Neo-Latin Epigram. A Learned and Witty Genre, Leuven University Press, 2009, p. 137-163 (surtout p. I45-152). Cette chercheuse a également consacré sa thèse de doctorat à l'œuvre poétique de Campano : Poetry and Patronage. Literary Strategies in the Poetry of Giannantonio Campano, dissertation inédite de l'université d'Amsterdam, 2007.

2. J. McManamon, Funeral Oratory and The Cultural Ideals of Italian Humanism, Chapel Hill, The University of north Carolina Press, I989, p. 52. Une récente contribution de C. Krebs a renouvelé l'étude du travail de l'humaniste comme ambassadeur pontifical après la mort de Pie II. Voir son Negotiatio Germaniae. Tacitus' Germania und Enea Silvio Piccolomini, Giannantonio Campano, Conrad Celtis und Heinrich Bebel, Göttingen, Vandenhoeck und Ruprecht, 2005.

3. P. Cecchini, Giannantonio Campano. Studi sulla produzione poetica, Urbino, I995, chapitre intitulé «L'editio princeps dei Carmina e le principali raccolte manoscritte », p. I2-36. - Nous citons les œuvres poétiques de Campano d'après l'édition procurée par I. B. Mencken, Epistulae et Poemata, Leipzig, Jean-Frédéric Gléditsch, 1707 .

4. F. Di Bernardo, Un vescovo umanista alla Corte Pontificia, Giannantonio Campano (I429-I477), Rome, Università Gregoriana editrice, "Miscellanea Historiae Pontificiae », 39, I975. 
dans lequel il perçoit la figure du pape victorieux béni de la Fortunes. Or, à ce constat fondamental, il convient d'ajouter que dans les pièces les plus importantes du corpus, Campano élabore la louange du pape à la manière traditionnelle des poètes courtisans. Il souligne notamment, lorsqu'il chante l'éloge de Piccolomini, le lien fort qui subsiste entre l'éloquence nourrie des muses et la victoire politique qui précède et conditionne le succès militaire.

À cette considération de l'image papale qui transparaît dans l'œuvre de Campano s'ajoute également une véritable poétique des genres. En effet, les poèmes les plus brefs, les épigrammes qui jalonnent les pages des Commentaires, tendent à privilégier l'aspect triomphal de la figure de Pie II. Leur brièveté, leur caractère monumental et épigraphique, n’autorisent pas le genre de réflexion nuancée nécessaire au poète qui cherchera souvent, dans les poèmes plus longs, à réconcilier des aspects apparemment contradictoires. C'est bien ainsi que l'ode lyrique destinée à Pie, comme aussi l'une des deux pièces en hendécasyllabes, présentent une louange du pape à l'occasion d'une festivité publique. Ces pièces révèlent aussi la même visée épigraphique, procédé fictionnel de l'éloge public, qui caractérise les épigrammes des Commentaires. En revanche, les longues élégies III, I et surtout IV, I esquissent un tableau plus nuancé de la personnalité du pape. Un homme juste, à la fois clément et rigoureux, capable d'une fermeté décisive au nom de la paix, telle est bien l'image de Pie II qui transparaît dans les vers de Campano. De ce pontife d'humbles origines, le poète dessine un portrait qui tient ensemble les qualités diverses qui font de lui, selon son humaniste protégé, à la fois un grand pape et un seigneur irréprochable.

\section{Les épigrammes des Commentaires}

La prose tardive de Pie II, notamment celle des célèbres Commentaires, contient plusieurs citations occasionnelles des vers de Campano qui viennent appuyer les propos du pape mémorialiste. Parfois ces vers couronnent la narration d'un événement auquel les distiques cités apportent un caractère solennel. Ainsi, au chapitre $26 \mathrm{du}$ cinquième livre des Commentaires, le prosateur cite une épigramme commémorant l'entrée que Pie II effectua à Tivoli le 3I juillet I46I. À cette occasion, le Pontife accordait son pardon à la ville qui s'était récemment soulevée contre l'autorité papale et fit ériger

5. S. De Beer, «Giannantonio Campano’s Poetry for Pius II Piccolomini », dans R. Di Paolo, A. Antoniutti et M. Gallo, Enea Silvio Piccolomini. Arte, Storia e Cultura nell'Europa di Pio II, Rome, Shakespeare and Company, 2, 2006, p. 307-322. 
une forteresse défensive dont la présence suscita des réactions bien diverses de la part des habitants de la ville. Un distique, vraisemblablement du pape lui-même, fut inscrit directement dans le marbre du portail, et à la description de cet appareil dans les Commentaires, le chroniqueur ajoute une épigramme de Campano. Cette pièce en cinq distiques souligne la clémence du saint-père - Esse Pium mitem nec tam punisse nocentes, v. 3. L'auteur note que l'esprit du pape qui s'abstient de punir les coupables est bien celui de la douceur et du pardon, alors que la perfidie des habitants de Tivoli (Tybur) lui paraît digne des origines grecques de cette ville :

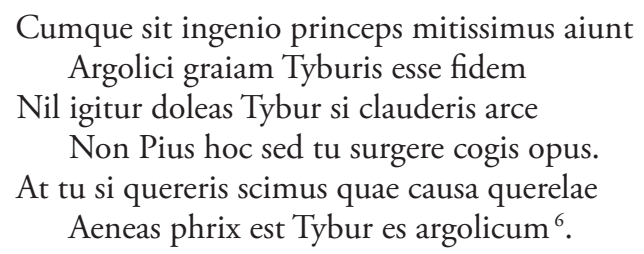

On peut souligner ici la remarquable consonance thématique de l'épigramme de Campano et de la prose de Piccolomini, qui déploie les mêmes termes lorsqu'il décrit ses propres gestes. La citation dans les Commentaires permet ainsi de mettre en évidence le degré auquel le travail de Campano relève d'un procédé de courtisan dont l'éloquence seconde les intérêts de son patron ${ }^{7}$. Le motif de la clémence de Pie II, central dans cette épigramme, constitue par ailleurs l'un des principaux thèmes récurrents des vers que l'humaniste rédigea en l'honneur du pape.

Une nouvelle citation d'épigramme clôture le chapitre I2 du septième livre des Commentaires. Dans ce passage, Pie II évoque les circonstances qui entourèrent l'un des grands événements publics de sa papauté. Il s'agit de la découverte d'une riche source d'alun, par le marchand et explorateur Giovanni da Castro, dans les collines près du village de Tolfa. Ici aussi, la citation de l'épigramme sert à commémorer un événement solennel, car une statue fut érigée près du lieu de la découverte, arborant la dédicace convenable : Johanni Castrensis aluminis inventori. Or, pour recentrer l'éloge sur la personne du pape lui-même, Campano souligne la bonne fortune du

6. Pie II, Commentarii, V, 26 : "Ils disent aussi que l'esprit du prince est très doux, / Mais que grecque est la foi de Tivoli argienne. / Ne te lamente pas, alors, Tivoli, si une roche t'enferme. / Ce n'était pas Pie, mais toi-même, qui l'as fait s'ériger. / Mais si tu te plains, nous saurons pourquoi : / Énée est phrygien, et toi, Tivoli, argienne. " - Toutes les citations des Commentaires proviennent de l'édition A. Van Heck: Pii II Commentarii rerum memorabilium que temporibus suis contigerunt, 2 vol., Città del Vaticano, Biblioteca Apostolica Vaticana, "Studi e Testi ", 3I3, I984.

7. Voir sur ce point les remarques de S. De Beer, «The Pointierung of Giannantonio Campano's Epigrams: Theory and Practice ", art. cité, p. I49. 
Pontife, déclarant que le royaume céleste n'est pas le seul domaine de la Création qui appartienne à la juridiction de Pie II. La terre aussi livre ses richesses à ce Pape auquel le destin sourit toujours :

Ipsa etiam, quod habet, tellus tibi contulit, et se

Praebuit eruptis prodiga visceribus.

Parte alia raucum aes, aliaque excussit alumen,

Datque alio argentum tertia vena loco $^{8}$.

Campano termine l'épigramme avec une pointe d'humour, en invitant la terre à révéler désormais ses trésors les plus convoités des hommes, à savoir ses veines d'or, pour éviter qu'on l'accuse de voler ce qui appartient de droit à Pie II :

Abdita telluris gremio haec latuere tot annos,

Et soli Domino nunc patuere suo.

Tu, quod nunc auri restat, ditissima tellus,

Ne furti rea sis, hoc quoque redde $\mathrm{Pio}^{9}$.

Ces vers légers soulignent avec esprit le rapport privilégié que le pape Piccolomini entretient avec le destin, selon la mythologie personnelle dessiné par le poète humaniste. Il s'agit en effet d'un thème central des éloges que Campano adresse à l'intention du pape.

Une troisième citation d'épigramme dans les Commentaires apparaît au début du livre IX, vers la fin de la somptueuse description que l'auteur consacre aux splendeurs du mont Amiata. Dans ce récit de voyage, Pie II note qu'il a déjà admiré le même endroit plusieurs fois auparavant, en raison de son amour sempiternel des lieux boisés (silvarum amator et varia videndi cupidus). C'est aussi cet amour de la nature, semble-t-il, qui a stimulé le mémorialiste à insérer une nouvelle épigramme dans son récit. "Le docte poète Campano, explique-t-il, élevé par Pie à la dignité épiscopale, par allusion aux guerres bien menées de Pie ainsi qu'à ses fréquentes retraites dans les forêts, composa les vers suivants ${ }^{10} \ldots$.. Cette épigramme ludique invente une prédestination au pape humaniste, déterminée par les prénoms de ses parents. Fils d'une mère Vittoria et d'un père Silvio, Pie II reçut dès la

8. Pie II, Commentarii, VII, I2, 3-6 : "La terre elle-même te livra ce qu'elle possède, et s'offre / Entière, prodigue, dans ses entrailles éclatées. / D'une part, elle produisit du bronze rauque; d'autre part, de l'alun; / Dans un troisième endroit, elle donne de l'argent. »

9. Pie II, Commentarii, VII, I2, 7-IO : "Ces trésors furent pendant tant d'années enfouis dans le sein de la terre, / Et parurent maintenant, uniquement pour leur Maître. / Toi, ô terre richissime, ce qu'il te reste maintenant d'or, / Donne-le aussi à Pie, pour que tu ne sois inculpé de vol. "

Io. Commentarii, IX, I, p. 519: "Campanus poeta doctus, quem Pius ad pontificalem honorem euexit, cum bella Pii cerneret felicia et frequentes secessus in siluas, huiusmodi uersus edidit. " 
naissance la prouesse guerrière ${ }^{\text {II }}$ et l'amour de la tranquillité des forêts ${ }^{\text {I2}}$. Ici encore, la poésie circonstancielle, citée avec approbation, esquisse un grand thème de l'éloge du Pape tel qu'il s'élabore sous le calame de Campano : Pie vainqueur, voyageur intrépide et vertueux chef de guerre.

Prises dans leur ensemble, les épigrammes de Campano qui apparaissent dans les Commentaires louent les qualités insignes que Campano attribue constamment au pape siennois lorsqu'il parle de lui. Le pontife humaniste se distingue notamment par sa prudence, sa clémence à l'égard même de ceux qui se sont montrés perfides et ingrats, le bonheur de l'homme élu par la Providence et par la Fortune, l'origine familiale qui l'a prédestiné à remporter les batailles et à aimer les forêts. Remarquable ici est l'inspiration résolument séculière de ces pièces de circonstance qui visent à rehausser l'éclat des gestes de Pie II. Dans ces épigrammes, Campano chante l'éloge de son bienfaiteur pontifical en des termes qui conviendraient également à la louange d'un aristocrate séculier ou même d'un condottiere. C'est qu'en célébrant divers aspects de la vie du pape, dans des pièces destinées à orner ses mémoires, le poète emprunte les modalités convenables au style même de Piccolomini qui parle de ses propres expériences dans une langue directe, précise et imagée. Il se peut également que certaines des épigrammes aient été adaptées ou remaniées lors des dernières corrections des Commentaires, effectuées par ce même Campano auquel l'auteur les avait confiés ${ }^{13}$.

\section{Une citation d'Enea Silvio : l'Oratio Cinericia}

Bien qu'elles aient été peu étudiées, individuellement, jusqu'à présent ${ }^{14}$, les épigrammes de Campano citées dans les Commentaires sont néanmoins bien connues de ceux qui étudient la prose de Pie II Piccolomini ${ }^{\text {Is. }}$. Moins

II. Pie II, Commentarii, IX, I, I-4 : "Quod victore Pio fieri tot praelia cernis, / Invalidasque suis hostibus esse manus, / Ne mirere, Pium peperit Victoria Mater; / Matris ab uberibus vincere sic didicit. " ("De ce que tu voies Pie vainqueur de tant de guerres, / Et que les bras de l'ennemi demeurent impuissants devant lui / Ne t'étonne pas, car à Pie donna naissance une Mère Victoire; / Aussi apprit-il depuis le sein maternel l'art de vaincre. ")

I2. Pie II, Commentarii, IX, I, 5-8: "Quod placeant sylvae, et magnum lustraverit orbem, / Sylvius hac genuit conditione Pater. / Jure igitur late spatiatur, et omnia vincit; / Patris obire orbem, vincere Matris habet. " "Que les forêts lui plaisent, et qu'il ait parcouru l'immensité de la terre, / Dans cette disposition Son Père Sylvius le créa. / De son Père il tient le goût du voyage, de sa Mère l'art de vaincre. ")

13. F. Di Bernardo, Un vescovo umanista alla Corte Pontificia..., ouvr. cité, p. 157-I69.

I4. Voir cependant S. De Beer, «The Pointierung of Giannantonio Campano’s Epigrams: Theory and Practice ", art. cité, p. I45 et suiv.

I5. F. R. Hausmann, «Untersuchungen zum neulateinischen EpigrammItaliens im Quattrocento », Humanistica Lovaniensia, 2I, I972, p. I-35. 
apparent sans doute est le fait que cette pratique de la citation chez Pie II s’inscrit dans un rapport de bons procédés qui, malgré la nature évidemment hiérarchique de leur relation, lie les deux poètes humanistes. Aussi peut-on remarquer que sur au moins une occasion importante, Campano se plaît à citer, dans sa propre prose latine, les vers de son confrère siennois amateur des Muses. C'est bien ainsi que, dans l'oraison qu'il prononce coram papa à Rome devant Pie II lui-même, vraisemblablement en $1463^{16}$, le nouvel évêque de Crotone insère deux distiques d'Enea Silvio qui datent de l'année I442, soit plus de vingt ans avant la prononciation du discours solennel. Dans ces vers, le jeune poète lauréat évoquait, au sein d'une réflexion sur l'immortalité de l'âme, l'épisode du vol du feu par Prométhée. Le remarquable "discours des Cendres " (Oratio cinericia), prononcé le premier jour du carême, élabore une réflexion sur l'origine de l'homme, au cours de laquelle Campano cite une abondance impressionnante de sources tant païennes que bibliques.

"Il ne fut jamais peuple ", déclare l'orateur devant la cour pontificale, " aussi ignorant, d'un genre de vie aussi barbare, qu'il ne s'enquit jamais de l'origine de l'homme $\mathrm{e}^{17}$ ». Au cours d'une démonstration érudite à caractère souvent éclectique, Campano évoque une diversité de réflexions parallèles, qui apparaissent chez Thalès, les Sages égyptiens et l'auteur de la Genèse, avant de citer enfin le mythe de Prométhée. Celui-ci constitue une illustration allégorique, analogue à la vérité chrétienne, dont la bonne interprétation doit corroborer la doctrine de l'église de Rome : "Mira vanitas fabularum. Sed quae si introspiciantur, videri possint non vanae ${ }^{18}$. "

L'étude rhétorique et thématique importante, que J. O'Malley a consacrée aux oraisons dominicales prononcées devant le pape, à Rome, dans la basilique de Saint-Pierre et à la Chapelle Sixtine, à l'époque qui s'étend de Nicolas V à Léon X (I45O-I529), montre que le corpus de cette éloquence sacrée est marqué par deux tendances divergentes ${ }^{19}$. Ces tendances prononcées permettent de diviser les discours en deux catégories, notamment

16. F. Di Bernardo, Un vescovo umanista alla Corte Pontificia..., ouvr. cité, p. I55 : "Nella Città Eterna il giovane presule tenne, nei primi mesi dell'anno, due discorsi che aumentarono discretamente la sua fama di oratore. Nel primo giorno di quaresima tenne l'Oratiocinericia davanti al papa e alla corte pontificia. Secondo il Ferni fu questo il primo incarico ufficiale che il Campano ricevette dal papa. "La datation de ce discours est l'objet de quelque hésitation de la part des érudits. Voir ainsi la note 37 à cette même page 155 chez Di Bernardo.

17. Joannis Antonii Campani Interamniensis Episcopi Oratio Cinericia. Romae dicta. Louvain, I548, p. 3 v $^{\circ}$ : "Multi quaesierunt originem hominis, nec fuit gens ulla tam ignara rerum, et victu barbara, quae non huius rei cognoscendae studio traheretur."

18. Joannis Antonii Campani Interamniensis Episcopi Oratio Cinericia..., ouvr. cité, p. 6 r ${ }^{\circ}$ : «Étonnante est la vanité des fables. Mais lorsqu'on les examine, elles peuvent ne pas paraitre frivoles. »

19. J. O'Malley, Praise and Blame in Renaissance Rome. Rhetoric, Doctrine and Reform in the Sacred Orators of the Papal Court, I450-I529, Durham, NC, Duke University Press, I979, p. I2 et passim. 
celles des oraisons «thématiques " et "épidictiques ». Les oraisons " thématiques " sont celles qui, appuyées par de véritables arguments qui sont renforcés de syllogismes, avancent la " preuve " d'un point de doctrine. En revanche, les oraisons dites "épidictiques " proposent, à la place d'une argumentation de type syllogistique, la somptueuse louange du Christ. Elles élaborent cette louange en déployant les ressources de langue et de figures héritées de la rhétorique ancienne. Le discours de Campano appartient de toute évidence à la deuxième catégorie, celle des oraisons épidictiques, comme en témoigne la forte prolifération de références à la littérature de l'Antiquité païenne, savamment parsemée de citations bibliques. Dans son évocation du mythe de Prométhée, Campano cite le début d'une élégie de Piccolomini lui-même, qui remonte à l'année même où l'humaniste de Sienne, trois ans avant d'entrer dans les ordres de l'Église, fut couronné poète lauréat par Frédéric III. Dédicacée au chancelier impérial Gaspard Slik, l'élégie contient 84 vers qui étalent brillamment l'érudition classique du jeune auteur ${ }^{20}$. L'orateur cite la poésie d'Enea Silvio pour rappeler à Pie II la légitimité morale des figurations allégoriques empruntées aux sources païennes :

Mira vanitas fabularum. Sed quae si introspiciantur, videri possint non vanae. Quod si petere a poetis liceret, ut paululum discederent a nugis, remque nobis nudam aperirent, quid dicturos aliud existimamus, nisi Prometheum opificem esse omnium rerum Deum? Minervam non Palladem Iovis natam cerebro, ut nugari solent, sed Dei sapientiam, eius menti insitam, atque infixam. Cuius ductu omnia fiunt, reguntur, gubernantur? Tum statuam corpus esse terrenum, Ignem clam coelo surreptum, animum esse nostrum e Dei sede profectum in corpus. Quod si negabis mihi Pater Sancte, afferam teipsum, qui id pereleganter expressisti versibus.

Non est animis mortalis origo.

Spiritus ex alta cuilibet arce datur.

Illinc Iapetides ignem ducente Minerva

Traxerat, ex illa vivimus usque face ${ }^{21}$.

20. Voir le texte complet dans Enee Silvii Piccolominei postea Pii PP. II Carmina, A. Van Heck (éd.), Città del Vaticano, Biblioteca Apostolica Vaticana, 1994, p. 65-69 (Epygrammata, XIV : Ad Gasparem Slik imperialem cancellarium).

2I. Joannis Antonii Campani Interamniensis Episcopi Oratio Cinericia..., ouvr. cité, p. $6 \mathrm{r}^{\circ}-\mathrm{v}^{\circ}:$ « Étonnante est la frivolité des fables. Mais lorsqu'on les examine de près, elles peuvent ne pas paraître frivoles. S’il était permis de chercher un exemple chez les poètes, quant ils s'éloignent quelque peu de leurs bagatelles, pour nous révéler la chose ouvertement, que pourrions-nous conclure d'autre, sinon que Prométhée est le Dieu créateur de toutes choses? Ce ne fut pas Pallas Minerve qui naquit du cerveau de Jupiter, comme le racontent vainement les fables, mais bien la sagesse de Dieu, située et enracinée dans son esprit. N'est-ce pas sous sa conduite que toute chose existe, que toute chose est réglée et gouvernée? Alors je suggérerai que notre corps est formé de terre et que le feu dérobé subrepticement au ciel est notre esprit, descendu depuis le siège de Dieu jusque dans notre corps. Si tu me nies cela, ô Saint-Père, je t'allèguerai toi-même, qui l'a exprimée très élégamment dans ces vers : "Les 
Les contextes des citations de vers dans la prose de Pie II et de Campano sont évidemment bien différents l'un de l'autre. Dans les Commentaires, les citations des épigrammes de Campano contribuent à une esthétique monumentale destinée à souligner la solennité des mémoires du pape. En revanche, la citation des vers du pontife lui-même, dans un important discours sacré que Campano prononça coram papa, souligne la légitimité d'un procédé intellectuel qui puise la bonne part de ses ressources dans un vaste corpus extérieur à la littérature biblique et patristique. Il s'agit là d'une manière de souligner la moralité profonde des écrits poétiques du jeune Piccolomini, pour lesquels il avait plus d'une fois subi des critiques, mais aussi de soutenir la validité des analogies mythiques fournies par la recherche humaniste. Malgré l'apparence toute païenne de leur écorce imagée, semble suggérer l'orateur, ces images une fois ramenées sous l'autorité du Créateur rehaussent sa gloire et peuvent même instruire les fidèles sur sa sublime grandeur.

\section{Les élégies encomiastiques}

Les épigrammes accueillies dans les Commentaires de Pie II livrent une première silhouette des poésies de Campano telles qu'elles pouvaient apparaître au regard de leur réception contemporaine. Ces mêmes œuvres, recueillies par Ferni et rééditées, corrigées, par Mencken en 1707, contiennent plusieurs poèmes destinés à l'intention de Pie II. En effet, si l'on inclut les deux épigrammes, de dix vers chacune, qui louent la ville de Pienza, cette partie du corpus poétique de Campano préservé dans les éditions posthumes compte au moins I4 pièces, aux formes et longueurs diverses. À cette liste, il convient d'ajouter aussi l'épigramme de quatre vers retrouvée par R. Bianchi dans le manuscrit Vatican latin 5167, intitulé «Ad pontem » et adressé à un détracteur de Pie $\mathrm{II}^{22}$. De ces I4 poèmes, une seule pièce fut rédigée en strophes saphiques, deux en hendécasyllabes et le reste en distiques élégiaques. La teneur encomiastique de ces poésies correspond de près à celle que l'on trouve déjà dans les épigrammes occasionnelles qui ornent la prose des Commentaires. La plupart de ces poésies brèves et ponctuelles, consacrées à la fonction encomiastique, ne permettent pas de reconstituer quelque portrait achevé de la figure pontificale. Or, les pièces

âmes ne sont pas d'origine mortelle. / C'est depuis une haute citadelle que chacun reçoit en don l'esprit. / De là, le fils de Japet, conduit par la Minerve, / Retira le feu; tous nous vivons de cette même étincelle." "

22. R. Bianchi, Intorno a Pio II : un mercante e tre poeti, Messina, Sicania, 1988, p. Io6. 
les plus longues, notamment les élégies d'occasion placées au début des livres III et IV des Carmina, apportent des couleurs à ce tableau conventionnel du pape justicier qui se distingue à la fois par sa prouesse et par sa clémence.

L'ode saphique intitulée "Ad Musas de Pio II " peut être qualifiée de pièce " héroïque " à l'instar de certaines œuvres de la lyre non seulement de Pindare et d'Horace, mais aussi de Filelfo, qui chantent l'honneur de princes et d'autres hommes puissants. Apollon et Minerve apparaissent, invoqués solennellement, dès la deuxième strophe :

\section{Nunc lyram, nunc profer Apollo carmen \\ Et poetarum decus eloquentum \\ Flava bellorum domitrix Minerva \\ Aegida pone ${ }^{23}$.}

L'attitude belliqueuse de la Minerve domitrix arborant son bouclier, annonce le caractère solennel de ce chant rédigé à l'occasion d'une festivité publique (haec spectacula) en son honneur. R. Bianchi a déjà bien souligné l'existence d'un florilège poétique en l'honneur du deuxième anniversaire de la papauté de Pie II. Il est raisonnable de croire que cette ode, et sans doute la pièce en hendécasyllabes qui la suit dans les Carmina, commémore le même événement festif. Un jeu sur le nom du héros célébré - "Venit Eneas Pius ô Camenae / Surgite " - confirme la pertinence référentielle de la culture de l'épopée guerrière. Campano évoque l'assemblement d'une latinité harmonieuse qui, désormais unie sous l'autorité papale, s'apprête enfin à mener la croisade contre la menace turque :

$$
\begin{aligned}
& \text { Iam strepet totum Latium sub armis, } \\
& \text { Undique illustres veniunt Tyranni } \\
& \text { Ad pedes nostri domini ferocem } \\
& \text { Turcum adituri }{ }^{24} \text {. }
\end{aligned}
$$

Campano énumère les forces européennes qui se rangent derrière le pape : le peuple d'Aquitaine (Ruteni), les Anglais (Britanni), les Cimbres (Cymbri) et les habitants de la Germanie (Germani) répondent tous à l'appel du souverain pontife. Les mêmes images, les mêmes motifs caractérisent également l'unique pièce en hendécasyllabes dédicacée nommément à Pie II. Ici, les noms d'Apollon, de Minerve, de Pius Aeneas apparaissent de nouveau.

23. Carmina, II, 22, dans Epistulae et Poemata, I.B. Mencken (éd.), ouvr. cité, p. II9 r ${ }^{\circ}$ : « Maintenant, Apollon, porte en avant ta lyre, ton chant / Et toi, honneur des poètes éloquents, / blonde Minerve dompteuse des guerres, / Pose ton bouclier."

24. Ibid., II, 22 : "Déjà le Latium tout entier bourdonne sous les armes, / De tous les coins du globe les rois célèbres viennent / Se rendre aux pieds de notre maître, prêts à partir / À la quête du Turc féroce. " 
Cette fois, néanmoins, le poète exhorte les muses à faire résonner des chants inconnus aux dieux antiques. Puis, à la fin de la pièce, il chante l'éloge de la Toscane qui fit jaillir les vers antiques nourris d'influences grecques, et qui, plus récemment, vit naître près de Sienne le saint-père de l'Église de Rome :

Felix Thusca plaga et beata terra,

Tu primum aonias referre musas

In nostram hesperiam, et fovere primum

Ausa es ${ }^{25} \ldots$

Dans ces deux poèmes, on le voit, la figure papale apparaît à travers deux thèmes connexes : le symbolisme conventionnel de la poésie païenne - celle des dieux inspirateurs - et la référence constante à l'initiative militaire pour la défense de la chrétienté. La pièce en hendécasyllabes ajoute l'éloge de la région toscane, patrie originaire en Italie de la translatio studii.

Cette double référence à la guerre et à la poésie imagée, constitue l'essentiel du discours encomiastique adressé à Pie II par son confrère et client originaire de l'Italie méridionale. Mais les pièces longues rédigées en distiques élégiaques y apportent une nuance considérable, puisque dans ces poèmes de circonstance importants, l'humaniste s'efforce de mieux décrire la nature belliqueuse du Pontife, afin de lui assigner des limites en la circonscrivant à l'intérieur d'un éthos fondé sur la clémence à l'égard des mortels. Ainsi, une élégie importante, intitulée Ad Pium Secundum Pontificem Maximum, ouvre le troisième livre des Carmina. Cette élégie, qui remonte vraisemblablement au stade primitif des relations entre Campano et Piccolomini, est d'un ton semblable à celui qui transparaît dans l'ode saphique et l'épigramme en hendécasyllabes présentées au pape lors d'une festivité publique en son honneur. Au début du poème, les Muses viennent se présenter au pontife humaniste qui pourtant les connaît déjà bien. Elles évoquent le long train de personnages qui viennent lui rendre hommage.

A travers cette démonstration de l'éloquence courtisane, l'auteur cherche évidemment à s'insinuer dans les bonnes grâces de son destinataire. Il emprunte notamment les motifs de l'élégie amoureuse, genre dans lequel Piccolomini s'était distingué avec un certain éclat bien avant sa carrière de Prince de l'Église. Un motif célèbre, propre à ce genre antique, est celui de la militia amoris, la milice d'Amour. Chez les Élégiaques antiques, ce motif repose sur l'opposition entre les souffrances inhérentes à la vie amoureuse et celles du soldat en campagne qui, souvent, passe la nuit entière couché

25. Ibid., II, 22, p. $119 \mathrm{v}^{\circ}$ : " Joyeuse région toscane et terre bienheureuse, / Ce fut toi qui la première osas ramener les muses / D’Aonie dans notre Italie, et, la première encore, tu osas / Les favoriser de caresses... " 
sur le sol. Selon Campano, Pie II, à la fois vainqueur et pacifique, est lui aussi un véritable soldat de l'amour. Projetant ainsi l'image du saint-père en homme de la paix et de la résolution diplomatique, l'humaniste lui fait remarquer que sa nature même l'éloigne des cruautés de la guerre.

Pie II, déclare le poète encomiastique, est un homme de l'écoute et de la conciliation. Cet homme chéri des saintes Muses, et dont la porte leur demeure toujours ouverte ${ }^{26}$, s'impose davantage par les moyens de l'éloquence, par la diplomatie de l'esprit, que par la force brutale des armes. Dans ces vers, même l'éloquence se présente à travers une métaphore guerrière :

Nos igitur, quoniam nulla est non gratia tecum,

Pro vate ad vatem nuntia turba sumus.

$\mathrm{Ne}$ vatis rideas nomen, tirunculus arma

Dum sequitur, certi militis instar habet ${ }^{27}$.

La césure de l'hexamètre au deuxième distique sépare deux thématiques distinctes et parallèles, celles notamment de la poésie et de la vie militaire, le poète débutant (ou moins distingué) ici caractérisé comme une nouvelle recrue (tirunculus). De cette accommodation un peu facile du motif militia amoris, Campano procède au développement qui concerne plus directement l'identité publique de Pie II. Le pontife humaniste, confirme le poète, suit les camps, non de Mars, ni de Vénus, mais bien des Muses :

Nos igitur tua castra sumus, tua proelia Musae,

$\mathrm{Si}$ quem nos tegimus, hic tibi miles erit ${ }^{28}$.

Il s'agit toujours pour Campano de suggérer que les poètes peuvent, auprès d'un tel pape, se réclamer du statut privilégié de confrères dans la culture des Muses. Cette affirmation banale conduit enfin l'humaniste à préciser l'attitude du pape à l'égard de la violence militaire :

Arma nihil tecum, belli furor omnis abesto :

$\mathrm{Ni}$ quod pro sancta relligione paras;

Hoc quo conficies animo studioque sagaci!

$\mathrm{O}$ quantum est animi, vincere consilio ${ }^{29}$ !

26. Ibid., III, I, p. I4I $\mathrm{r}^{\circ}$ : "Scit bene, quam pateat sacris haec janua Musis, / Et timeat sanctas nulla repulsa fores."

27. Ibid., III, I : " Nous sommes alors, puisque rien n'est sans grâce auprès de toi, / La troupe messagère d'un poète à l'autre. / Et ne ris pas de ce nom de poète, car lorsqu'une recrue nouvelle / Suit les armes, elle ne ressemble pas moins à un soldat chevronné. "

28. Ibid., III, I : « Nous sommes ton armée en effet, nous les Muses, / Si nous protégeons quelqu’un, celui-là sera ton soldat. "

29. Ibid., III, I, p. I4I $\mathrm{v}^{\circ}:$ « Aucune place aux armes près de toi - qu'elle demeure lointaine, toute fureur guerrière / Autre que celle que tu prépares au nom de la sainte religion; / Celle-ci, tu l'organises avec une si grande 
Comme preuve, il évoque encore l'exemple récent (nuper) du conflit à Assisi, apaisé par Pie II sans le moindre recours aux armes ${ }^{30}$. Tout en alléguant cet exemple d'une victoire remportée par la diplomatie et la clémence de l'homme d'Église, il laisse néanmoins ouverte la possibilité que le saintpère déploiera la force militaire lorsqu'il sera appelé à protéger les principes et les intérêts de la Religion même : "Ni quod pro sancta relligione paras..." Le motif de l'arme de l'éloquence apparaît de nouveau, dans une forme bien plus élaborée, dans l'élégie de 364 vers qui ouvre le quatrième livre des Carmina. Cette pièce importante, véritable récapitulation de la carrière du poète destinée au souverain pontife ${ }^{31}$, est vraisemblablement plus tardive que l'élégie III, I. En effet, lorsqu'il écrit cette longue élégie, Campano brigue activement les fonctions épiscopales qu'il assumera en I462. Ici, le poète humaniste revisite les thèmes, parallèles et complémentaires, de la prouesse guerrière et de la clémence de Pie II. Il se plaît à souligner notamment le pouvoir universel du pape :

Quippe tuum quod habet totum sub nubila coelum est, Juraque totius nunc Pius orbis habet ${ }^{32}$.

À partir de ces motifs conventionnels, Campano dresse le portrait de Pie II comme diplomate suprême et pacifiste convaincu, dont le pouvoir s'érige sur un fonds de principes et d'éloquence. Or, l'homme dont l'arme principale est celle de l'éloquence, se doit d'accorder aux lettrés de son entourage la liberté qui leur permet de nourrir la mielleuse faconde des muses. Campano décrit à nouveau dans cette élégie la séduisante prolixité de son maître, déployant cette fois une métaphore plus vivace. Les paroles de Pie II sont non seulement susceptibles de convaincre par la clarté rigoureuse de la générosité et de l'amour de l'autre qui les inspire, mais aussi par l'effet d'émerveillement lumineux qu'elles induisent toujours dans l'esprit de l'auditeur :

Proelia dum suades facili mortalibus ore,

Adversus Turcas expedienda manus;

Non tantum hostis atrox, quantum tua lingua movetur,

Nescio quid graviter dijaculata potest.

sagesse de cœur et d'effort! / Ô que c'est beau de remporter la victoire par la prudence de l'esprit! » 30. Ibid., III, I : « Nuper ut Assisium nullo tibi Marte subactum est, / Magna, sed ingenio gloria digna tuo." 31. F. Di Bernardo, Un vescovo umanista alla Corte Pontificia..., ouvr. cité, p. I66: "Questi versi, tratti da una lunga elegia, furono scritti quando Pio aveva già manifestato il progetto di condurre la guerra contro i turchi. Ciò acquista un significato particolare, perché la lunga composizione poetica rappresenta un bilancio di tutta l'attività letteraria precedente."

32. Carmina, IV, I, p. I89 v ${ }^{\circ}$, ouvr. cité : " Tout ce qui apparaît sous les nues du ciel t’appartient en effet, / Et Pie dispose maintenant des lois de l'univers entier. " 
Spicula torsist nostris non nota pharetris,

Qualia nec prisca torta fuere manu.

Haesimus attoniti, qualis qui tactus ab igni est

Aethereo, quo sit, non vide tille, loco.

Ense gerant alii, moveas tu proelia lingua :

Ut bene movisti, sic bene mota geras ${ }^{33}$.

Le poète humaniste ne se contente pas de souligner l'alternative sempiternelle qui subsiste entre ces deux manières de conduire la guerre contre les ennemis de la foi. Il développe la métaphore de manière à montrer l'équivalence de l'épée et de la plume:

Dux acer vestigat equos, vestigat et arma;

Quaerit quem telis, quem quoque donet equo.

Praefectos legit ille bonos, legit ille Tribunos

Ignavus laudum nil ibi miles habet ${ }^{34}$.

Le soldat dont il est ici question, c'est bien l'humaniste lui-même, Campano, qui se propose, suivant la convention des poètes courtisans, d'écrire une épopée en l'honneur des exploits de Pie II. Sous le patronage de son maître pontifical, la " nouvelle recrue » de l'élégie III, I s'est métamorphosée en poète épique. Mais il s'agit là, bien entendu, d'un nouveau motif élégiaque, celui du poète "gracile " qui affiche son désir d'abandonner le genre mineur qu'est l'élégie afin de chanter des exploits de guerre ${ }^{35}$.

Autour de Pie II, on le voit, le motif de la guerre suscite une réflexion sur l'équilibre entre les vertus humaines de la clémence et la virtù héroïque du guerrier. Les pièces les plus brèves, les épigrammes, tendent à privilégier soit l'une, soit l'autre de ces deux qualités. Dans ce même esprit, les vers saphiques et les hendécasyllabes présentent un discours triomphal, un lyrisme d'allure " officielle » et publique, qui chante la gloire seigneuriale de Pie II, défenseur éclairé de la Chrétienté. Seule la forme élégiaque, le long poème en distiques, permet au poète humaniste de nuancer le portrait général du pontife qui émerge de l'ensemble de ses vers. Le ton personnel, la révélation autobiographique, des deux longues élégies III, I

33. Ibid., IV, I, p. $192 \mathrm{r}^{\circ}$ : "Alors que d'une bouche persuasive tu conseilles aux mortels / De porter la guerre contre les forces des Turcs, / L’ennemi n'est pas autant féroce, que ta langue est agile, / Qui se déploie avec je ne sais quelle énergie. / Des javelots tu lanças inconnus de nos carquois, / Qui ne furent jamais lancés par les mains anciennes. / Nous nous arrêtâmes, émerveillés, tel l'homme frappé par le feu / Céleste, qui ne sait plus où il se trouve. / Que d'autres portent l'épée, ta part est de mener des batailles avec la langue diserte. / Selon que tu as bien parlé, ainsi sauras-tu mener la guerre. »

34. Ibid., IV, I, p. $192 \mathrm{v}^{\circ}$ : « Le chef ardent se procure les chevaux, il se procure les armes, / Il cherche à savoir qui doit porter la lance, qui doit monter à cheval. / Celui-là choisit les bons commandants, les bons Tribuns; / Pas de salaire, chez lui, pour le soldat indifférent à la gloire. "

35. Properce, Elegiae, II, ro. 
et IV, I, leur prêtent aussi un caractère épistolaire qui favorise l'intimité de la voix qui parle. Ici, dans des vers de circonstance, l'humaniste courtisan se présente, se livre, s'explique. Aussi s'efforce-t-il de conduire la double thématique de la guerre et de la clémence à une convergence cohérente et digne de la figure du Pape. Il emprunte la voie traditionnelle, familière aux humanistes, de la métaphore qui réunit la prudence clémente et la prouesse martiale sous l'égide de l'éloquence, arme des rhéteurs mais aussi des guerriers éclairés, tels Nestor, Ulysse, Énée, dont la tradition épique fut si féconde. Cette initiative suprême situe aussi le poète Campano près des limites extrêmes du genre élégiaque ${ }^{36}$, comme il le laisse entendre luimême dans la grande élégie qui ouvre le quatrième livre de ses Carmina. Dans la mesure où il s'efforce de restituer une certaine image juste et réelle de Pie II, le poète revient vers un registre narratif apte à tresser ensemble les diverses références occasionnelles qui sont la matière même de la poésie encomiastique. Ce fonds narratif, qui transparaît dans les poésies longues, permet à l'humaniste d'esquisser le profil d'un personnage proprement héroïque, qui possède toutes les complexités d'un homme à la fois exemplaire et profondément humain.

36. La critique a déjà bien souligné le caractère inventif, et souvent novateur, des élégies de Campano. Voir notamment l'excellent article de J.-L. Charlet, "L'âge d'or dans une élégie de Giannantonio Campano (Carm. II, 9) ", Respublica Litterarum, 22, I999, p. I28-I38. 